\title{
Ungleichheit in globalen Wertschöpfungsketten: eine intersektionale Perspektive auf soziale Reproduktion
}

\author{
Martina Sproll
}

(C) Der/die Autor(en) 2020

Zusammenfassung Der Artikel fragt nach der Bedeutung von sozialer Ungleichheit für die Dynamik globaler Produktion. Trotz einer breiten feministischen Debatte zur Rolle von Geschlechterverhältnissen in globalen Wertschöpfungsketten seit den 1970er-Jahren wurde diese Dimension systematisch aus dem Mainstream der weiteren Theorieentwicklung ausgeblendet. Der vorliegende Beitrag schärft den Blick für soziale Ungleichheit in transnationalen Produktionsnetzen, indem zum einen die Verflechtung von bezahlter und unbezahlter sowie formeller und informeller Arbeit beleuchtet wird, die immer auch geschlechtlich strukturiert ist. Zum anderen wird die Bedeutung privater Haushalte für die Aneignung von Mehrwert durch (transnationale) Unternehmen thematisiert, die den Zusammenhang von produktiver und reproduktiver Sphäre sichtbar macht. Akkumulation in globalen Wertschöpfungsketten basiert auf der gezielten Ausnutzung von Ungleichheit und Diskriminierung, die auf der Verflechtung unterschiedlicher Ungleichheitsdimensionen wie Geschlecht, Ethnizität/Race, Klassen- bzw. Kastenzugehörigkeit, Alter oder Region beruhen. Eine intersektionale Perspektive auf Ungleichheit ist deshalb unabdingbar, um die komplexen Macht- und Herrschaftsverhältnisse zu verstehen und entsprechende Gegenmaßnahmen entwickeln zu können.

Schlüsselwörter Globale Wertschöpfungsketten · Soziale Ungleichheit · Geschlechterverhältnisse · Intersektionale Ungleichheit · Soziale Reproduktion

\footnotetext{
M. Sproll ( $\bowtie)$

Fachbereich Wirtschaftswissenschaften, Hochschule für Wirtschaft und Recht Berlin, Badensche Straße 52, 10825 Berlin, Deutschland

E-Mail: martina.sproll@hwr-berlin.de
} 


\title{
Social inequalities in global value chains: an intersectional perspective on social reproduction
}

\begin{abstract}
The article focuses on the significance of social inequality for the dynamics of global production. Despite a broad feminist debate on the role of gender relations in global value chains since the 1970 s, this dimension has systematically been excluded in the mainstream theory development. The present contribution sharpens the focus on social inequality in transnational production networks by, on the one hand, illuminating the interdependence of paid and unpaid as well as formal and informal work, which is always gendered. On the other hand, the significance of private households for the appropriation of hidden surplus by (transnational) companies is addressed, thus illuminating the connection between the productive and reproductive spheres. Accumulation in global value chains is based on the exploitation of different dimensions of inequality such as gender, ethnicity/race, class/caste, age or region. An intersectional perspective on inequality is therefore indispensable for understanding the complex power relations and for developing appropriate countermeasures.
\end{abstract}

Keywords Global value chains - Social inequalities · Gender relations · Intersectional inequalities $\cdot$ Social reproduction

Die Globalisierung von Arbeit und Produktion hat breite wissenschaftliche Debatten und neue theoretische Konzepte mit unterschiedlicher Schwerpunktsetzung hervorgebracht. Im Zuge der zunehmenden Internationalisierung von Arbeit und Produktion entstanden bereits in den 1970er-Jahren viele „Weltmarktfabriken“ in Exportproduktions- bzw. Freihandelszonen insbesondere in Lateinamerika und Asien. Sie zogen das Interesse vieler WissenschaftlerInnen auf sich, die sich u. a. mit der Entstehung einer „Neuen Internationalen Arbeitsteilung“ beschäftigten (Fröbel et al. 1977). Es ging um die Frage, welche Arbeits- und Produktionsprozesse durch die Verlagerung aus den zentralen Industrieländern in die Peripherie entstanden, welche Beschäftigungsverhältnisse vorherrschten und auch, wer die Beschäftigten waren in großer Mehrheit junge Frauen, die als billige Arbeitskräfte in globale Produktionsverhältnisse integriert wurden. Bereits in dieser Zeit entstanden die ersten Forschungsarbeiten über die Bedeutung von Geschlechterverhältnissen in der globalen Produktion (z. B. Nash und Fernandez-Kelly 1983). Sie knüpften zum Teil unmittelbar an dependenz- und weltsystemtheoretische Ansätze an und machten sichtbar, dass transnationale Produktion auch mit neuen Formen der geschlechtlichen Arbeitsteilung und der Ausbeutung von geschlechtlicher Ungleichheit verbunden waren. Leider wurde die umfang- und erkenntnisreiche feministische Forschung in der weiteren Theorieentwicklung in den Konzepten zu „Global Commodity Chains“ oder ,Global Value Chains“ zumindest in den Mainstream-Debatten kaum aufgegriffen. Vielmehr ist eine starke Verengung auch der früheren weltsystemtheoretischen Ansätze auf industrielle Konstellationen und betriebliche Dynamiken zu beobachten, sodass soziale Ungleichheit bezogen auf Geschlecht und andere Dimensionen ausgeblendet blieb (kritisch Bair 2010; Collins 2014). 
In diesem Artikel soll deshalb mit Bezug auf vorliegende Forschungsliteratur und Debatten der Blick für soziale Ungleichheiten in transnationalen Produktionsnetzen geschärft werden. Ohne eine solche Perspektive kann die Dynamik globaler kapitalistischer Akkumulation mit ihren komplexen Macht- und Herrschaftsverhältnissen sowie ihren sozialen und kulturellen Normen nicht vollständig gesehen und verstanden werden. Soziale Ungleichheit ist hierbei zum einen als analytische Kategorie zu verstehen, die immer noch weitgehend als ,missing link“ in der Theoriedebatte erscheint. Gleichzeitig kann auch die Entwicklung von Politikmaßnahmen gegen Ausbeutung und Diskriminierung nicht vollständig und sinnvoll gelingen, wenn die Unterschiede zwischen verschiedenen Beschäftigtengruppen, ihr soziales Profil, ihre Machtressourcen usw., nicht einbezogen werden - sei es aus der Perspektive internationaler Organisationen, sei es aus der Perspektive der Gewerkschaften oder lokaler Nichtregierungsorganisationen. Es reicht also nicht aus, nur die Dimension Geschlecht zu adressieren. Vielmehr ist es längst common sense in der kritischen feministischen Literatur, dass diese immer verflochten mit anderen Ungleichheitsdimensionen, also als eine intersektionale Ungleichheit verstanden werden muss (Roth 2019). So haben auch andere Kategorien von Ungleichheit eine strukturierende Bedeutung für die Form und Gestaltung von globaler Wertschöpfung, wie insbesondere Diskriminierung basierend auf Race/Ethnizität und der sozialen Herkunft, aber auch Region, Alter oder sexuelle Orientierung.

Eine weitere Ebene, die hier in die Analyse einbezogen wird, ist die Bedeutung informeller Arbeit. Die meisten Studien, die in der Forschung zu globalen Wertschöpfungsketten entstanden sind, fokussieren fast ausschließlich auf formale Beschäftigungsverhältnisse in den Unternehmen, doch Informalität ist gleichwohl strukturierend für die Dynamik globaler Produktion und damit verbundene Ungleichheitsprozesse und von grundlegender Bedeutung an vielen Schnittstellen nicht nur am „Ende der Kette“. Dabei spielen - und dies ist insbesondere in der feministischen Debatte immer wieder betont worden - auch die privaten Haushalte eine zentrale Rolle. Sie sind zum einen selbst Produktionsort und zum anderen fließt die unbezahlte Reproduktionsarbeit, welche überwiegend von Frauen geleistet wird, in die Wertschöpfung ein. So geht die Entwicklung einer spezifischen Form kapitalistischer Akkumulation immer auch mit einer Formveränderung der sozialen Reproduktion einher und umgekehrt. Dies verweist zugleich auf grundlegende gesellschaftliche Veränderungen und die entsprechende Institutionalisierung durch staatliche Politiken.

\section{Commodity Chains und die soziale Reproduktion}

Der Begriff der „Global Commodity Chains“, also der globalen Waren- oder Güterketten, wurde von vielen WissenschaftlerInnen unterschiedlicher Disziplinen benutzt, um die Entwicklung globaler Produktion seit den 1990er-Jahren zu analysieren. Der Vorteil des Konzepts liege in seiner Fähigkeit, ökonomische Globalisierung auf die Alltagspraxen von Firmen, Beschäftigten, Haushalten, Staaten und KonsumentInnen anzuwenden, so Bair und Werner (2011, S. 988). Ursprünglich geprägt 
wurde der Begriff der „,commodity chains“ von Terence Hopkins und Immanuel Wallerstein, die ihn in den späten 1970er-Jahren folgendermaßen definierten:

What we mean by such chains is the following: take an ultimate consumable item and trace back the set of inputs that culminated in this item - the prior transformations, the raw materials, the transportation mechanisms, the labor input into each of the material processes, the food inputs into the labor. This linked set of processes we call a commodity chain. If the ultimate consumables were, say, clothing, the chain would include the manufacture of cloth, the yarn, etc., the cultivation of the cotton, as well as the reproduction of the labor forces involved in these productive activities (Hopkins und Wallerstein 1977, S. 128).

In diesem berühmten Zitat versuchten Hopkins und Wallerstein das Konzept der commodity chains breit und in der Perspektive des Weltsystem-Ansatzes zu fassen. Die Herstellung eines Produkts basiert hier auf der raum-zeitlichen Vernetzung von unterschiedlichen Prozessen, Stoffen und AkteurInnen. Wirklich bemerkenswert ist aber, dass nicht nur die Produktion, sondern auch die daran beteiligten Arbeitskräfte hier explizit erwähnt werden - somit geht dieser Ansatz weit über die ökonomischen Analysen des Mainstreams in der Güterkettenforschung hinaus. Die Frage der sozialen Reproduktion - die Arbeitskräfte, ihre häuslichen und lebensweltlichen Bedingungen wie etwa Wohnen, Ernährung usw. zur Aufrechterhaltung und Reproduktion der Arbeitskraft - erscheint hier vielmehr als inhärenter Bestandteil des Konzepts. Dunaway fasst in ihrem Buch „Gendered Commodity Chains“ diesen Ansatz folgendermaßen zusammen: Hopkins und Wallerstein seien davon ausgegangen, dass commodity chains auf Sexismus, Rassismus und der Aneignung des in privaten Haushalten erwirtschafteten Mehrwerts beruhten (Dunaway 2014a, S. 2). Basierend auf mehreren Publikationen aus den 1980er- und 1990er-Jahren ließen sich ihre Hauptpunkte diesbezüglich - und damit die grundlegende Charakteristik von commodity chains - folgendermaßen darstellen:

1. Die Verflechtung verschiedener Formen von bezahlter und unbezahlter, freier und unfreier Arbeit;

2. Ausbeutung und Aneignung des sichtbaren wie auch des versteckten Mehrwerts aus reproduktiver bzw. haushaltsbasierter Arbeit;

3. Ausbeutungsverhältnisse, die auf der Zugehörigkeit zu Geschlecht oder Race/ Ethnizität beruhen;

4. die ökonomische Abwertung der Hausarbeit (ebd., S. 2).

Damit sind alle wichtigen Dimensionen benannt, die für eine Perspektive auf Macht und Ungleichheit in kapitalistischen Akkumulationsprozessen notwendig sind, sofern man diese als soziales Verhältnis bzw. als Ausbeutungsverhältnis begreift. Dieses umfassende Konzept wurde jedoch nicht stilbildend für die Analyse transnationaler Produktionsverhältnisse. Die Dimension Geschlecht und noch viel mehr die Bedeutung privater Haushalte wurden zunehmend aus den Analysen ausgeblendet. 


\section{Neue Internationale Arbeitsteilung - Gender in der globalen Produktion}

Spätestens in den 1970er-Jahren setzte eine neue Welle der Globalisierung von Produktion ein, die für die genannten Dimensionen von commodity chains einen neuen Strukturzusammenhang bildet und ihre Bedeutung intensiviert. Wie eingangs erwähnt, entstand bereits in dieser Zeit umfangreiche Forschung zu Geschlecht in der transnationalen Produktion. In der Folge der Krise des Fordismus kam es in den 1970er-Jahren zu einer massiven Verlagerung von Produktionsprozessen in die Peripherie, insbesondere nach Asien und Lateinamerika. Fröbel et al. (1977) haben diese Entwicklung mit ihrer Theorie der „Neuen Internationalen Arbeitsteilung“ beschrieben. Demnach verblieben in dieser Phase die kapitalintensiven Prozesse in den alten Industrieländern, während die arbeitsintensiven Prozesse sozusagen als ,,verlängerte Werkbank" der alten Standorte in die Peripherie verschoben wurden, also in Regionen mit einem großen Angebot billiger und niedrig qualifizierter Arbeitskräfte, die vorwiegend in der noch wenig automatisierten Textil- und Bekleidungs- sowie der Elektro- und Elektronikproduktion eingesetzt wurden (ebd., S. 149, siehe auch Sproll 2016a). Kennzeichnend für die Arbeitsorganisation waren tayloristisch organisierte Produktionslinien, die auf die Ausbeutung niedrig qualifizierter, niedrig bezahlter und kaum gewerkschaftlich organisierter Arbeitskräfte zielten, sodass auch von ,blutigem Taylorismus“ gesprochen wurde (Lipietz 1987). Und: Es war auffallend, dass die meisten dieser Arbeitskräfte junge Frauen waren, die bislang keine Erfahrung und Sozialisation als Lohnarbeiterinnen hatten. Die Forschung lenkte den Blick auf die Ausbeutung dieser weiblichen Arbeitskräfte insbesondere in der Textil- und Elektronikindustrie. Zu nennen sind etwa die Studien von Fernández-Kelly (1983) zur Maquiladora-Industrie in Mexiko, Ong (1987) zu den neuen Produktionszonen in Asien und dem Widerstand der weiblichen Arbeitskräfte oder der vielbeachtete Aufsatz „Nimble Fingers Make Cheap Workers“ von Elson und Pearson (1981), der nicht zuletzt die Bedeutung der Geschlechter-Konstruktionen (,fügsam und mit flinken Fingern“) für die Ausbeutung junger Frauen in den Weltmarktfabriken aufzeigte. Die Studien trugen dazu bei, die Geschlechter-Dimension in der Globalisierung von Arbeit und Produktion zu thematisieren, indem sie u. a. zeigten, dass Frauen dabei auch im globalen Süden aus traditionellen Formen der geschlechtlichen Arbeitsteilung herausgelöst und in formale Erwerbsarbeitsverhältnisse integriert wurden dass also die globale Ausdehnung kapitalistischer Produktion auch eine Landnahme der privaten Haushalte und der Arbeitskraft von Frauen bedeutet. Dies macht auch deutlich, dass soziale und kulturelle Normen, die Organisation der sozialen Reproduktion und geschlechtliche Macht- und Herrschaftsverhältnisse unmittelbar als Teil der Globalisierung von Arbeit und Produktion zu verstehen sind, wie es Maria Mies in ihrem Buch „Patriarchat und Kapital - Frauen im globalisierten Kapitalismus“ bereits 1986 zur Diskussion stellte (Mies 2014). 


\section{Von Global Commodity zu Global Value Chains und Production Networks}

Seit den 1990er-Jahren setzte eine neue Welle der Globalisierung von Arbeit und Produktion ein, die auf der neoliberalen Öffnung und Deregulierung von Märkten beruht. Damit wurde eine neue Phase finanzgetriebener kapitalistischer Akkumulation eingeläutet, die sich durch Flexibilisierung und neue Formen der transnationalen Verflechtung von Produktions- und Arbeitsbeziehungen auszeichnet. Die daraus folgende wissenschaftliche Debatte generierte neue theoretische Konzepte, insbesondere zu „Global Commodity Chains“ (GCCs) und „Global Value Chains“ (GVCs) (z.B. Gereffi und Korzeniewicz 1994; Gereffi und Kaplinski 2001). In diesen Konzepten ging es vor allem darum, die komplexen Beziehungen zwischen einzelnen Unternehmen, Zulieferern, Subunternehmen etc. zu analysieren und zu verstehen, wie diese Ketten eigentlich gesteuert werden bzw. von wem - also wer hat warum die Macht in der Kette, sich z.B. den erwirtschafteten Mehrwert anzueignen. Mit ihrem Ansatz der Global Commodity Chains versuchte die Gruppe um Gary Gereffi zunächst eine Weiterentwicklung der weltsystemtheoretisch ausgerichteten Ansätze durch die Unterscheidung verschiedener Typen von Wertschöpfungsketten zu leisten (z.B. von sogenannten ,,buyer-driven“ versus ,,producer-driven commodity chains“, Gereffi 1996). Dabei galt das Interesse vor allem der Frage des ,industrial upgrading“, also einer industriellen Höherentwicklung in sogenannten „Entwicklungsländern“. Es wurde deutlich, dass diese neue Phase der Globalisierung nicht mehr den Mustern folgte, die Fröbel, Heinrichs und Kreye in ihrer „Neuen Internationalen Arbeitsteilung“ identifiziert haben. Es ging nicht mehr um die ,,verlängerte Werkbank", sondern zunehmend um die Verlagerung hochqualifizierter ProduktionsInfrastrukturen in die Peripherie (Lüthje et al. 2013). Es stellte sich also die Frage, ob die Integration in GCCs „Entwicklungsländer“ a) in eine wettbewerbsfähigere Position auf dem Weltmarkt bringen würde und b), ob diese im Zuge des industrial upgrading - also der Entwicklung höherwertiger Produkte, die auch höhere Qualifikationen von den Beschäftigten erfordern und höhere Löhne nach sich ziehen - auch der Bevölkerung zu mehr Wohlstand verhelfen würde (Gereffi 1999; Humphrey und Schmitz 2002).

Damit brach dieses Konzept mit der Tradition des Weltsystem-Ansatzes, der strukturelle Asymmetrien im Weltsystem in den Vordergrund stellte. Bair zufolge hat der GCCs-Ansatz eher den Fokus einer netzwerkbasierten Organisationsanalyse eingenommen und die Aufmerksamkeit auf unterschiedliche Formen der Governance gelenkt (2005, S. 158 ff.). Bei allem Erkenntniszugewinn, der durch die vielen Studien mit dem Konzept der GCCs sicherlich gelang, ist aber die oben diskutierte holistische Perspektive des Weltsystem-Ansatzes, der auch die soziale Reproduktion umfasste, auf der Strecke geblieben. Das heißt insbesondere, dass die Bedeutung der Geschlechterverhältnisse, aber auch andere Dimensionen sozialer Ungleichheit wieder aus der Aufmerksamkeit der wissenschaftlichen Forschung verdrängt wurden.

In der theoretischen Weiterentwicklung wurde der GCCs-Ansatz vom Konzept der Global Value Chains weitgehend abgelöst. Diesem bescheinigt Bair jedoch noch mehr als dem Vorgänger-Konzept eine Engführung der Perspektive auf die MesoEbene der Branchen-Dynamik und auf das industrial upgrading auf der Mikroebene 
(ebd., S. 153). Collins geht in der Rekonstruktion, wie soziale Ungleichheit, Geschlecht und soziale Reproduktion aus den theoretischen Konzepten verschwunden sind, noch einen Schritt weiter. Sie beschreibt, wie die Gruppe um Gereffi um die Jahrtausendwende das Ziel verfolgte, die Analysemethoden stärker zu standardisieren und an traditionelle volks- und betriebswirtschaftliche Analysen anzupassen, um damit größere Schärfe in der Analyse und eine höhere Relevanz für die Entwicklung von Policy-Programmen zu erreichen. Dafür sei es notwendig, von einer stark heterogenen empirischen Evidenz globaler Produktion zu abstrahieren und Komplexität zu vereinfachen (Collins 2014, S. 30). Collins sieht in dem engen Fokus auf Wettbewerbsfähigkeit und industrielle Höherentwicklung eine Annäherung an neoklassische Paradigmen mit dem Resultat, dass die Menschen aus dem Untersuchungsfeld verschwunden seien - somit seien auch die Perspektiven für eine Theorieentwicklung, die den geschlechtlichen und rassistischen Hintergrund für die Aneignung von verstecktem Mehrwert in den Blick nehmen, systematisch eingeengt bzw. ausgeschlossen worden (ebd., S. $30 \mathrm{f}$.).

Natürlich ist die Debatte vielfältig und inzwischen sehr ausdifferenziert. An den vorgestellten Konzepten gab es viel Kritik, aber auch Weiterentwicklung. So wurde z.B. von einer Gruppe aus dem industriegeografischen Kontext an der Universität Manchester das Konzept der Global Production Networks (GPN) geprägt (Henderson et al. 2002). Hier wurde z.B. die Rolle des Staats oder auch die Bedeutung von räumlicher Verteilung (space) viel stärker in den Blick genommen - wo und warum siedeln sich Unternehmen eigentlich an und inwieweit ist das in einen historischen, ökonomischen, politischen, sozialen und kulturellen Kontext einzubetten? In diesem Ansatz wurden also Machtverhältnisse viel grundlegender thematisiert. Dennoch bedeutete dies nicht, dass nun Geschlecht und soziale Ungleichheit bzw. soziale Reproduktion wieder systematisch einbezogen worden wären.

Zusammenfassend lässt sich festhalten, dass die Erkenntnisse der Genderforschung nicht grundlegend oder nur verkürzt in die konzeptionelle Entwicklung zu globalen Wertschöpfungsketten integriert wurden. Interessant - und anschlussfähig an die Geschlechter- bzw. Ungleichheitsforschung - ist jedoch, dass zunehmend das Thema „Arbeit“ in den Fokus der Analysen genommen wurde (Selwyn 2012). Dies erlaubt zum einen, die Beschäftigten nicht nur als Produktionsfaktor, sondern als AkteurInnen zu sehen, die die Form globaler Produktionsnetze aktiv durch (kollektiven) Widerstand und Verhandlungen mitgestalten. Zum anderen rückte auch die Frage nach den Beschäftigungsverhältnissen in den Mittelpunkt, die insbesondere unter dem Label des Programms ,,decent work“ der Internationalen Arbeitsorganisation (ILO) firmieren, in dem u. a. menschenwürdige Arbeit in globalen Wertschöpfungsketten reklamiert wird (ILO 2016). Die Forderung nach „,bringing labour back in“ erlaubte es, die Aufmerksamkeit wieder auf den Arbeitsprozess zu richten (wie es in den Arbeiten zur Neuen Internationalen Arbeitsteilung schon der Fall war), sodass hier Anschlüsse zur Labour Process Debate hergestellt (siehe z. B. Taylor et al. 2013) und wichtige Rückschlüsse über die Produktion von Ungleichheit gewonnen werden konnten (Sproll 2010). 


\section{Neuere Entwicklung der Geschlechterforschung im Kontext globaler Wertschöpfung}

Nun hat es seit den 1990er-Jahren auch weiterhin wichtige Studien zu Gender in globalen Wertschöpfungsketten gegeben, die sich außerdem ausdifferenziert haben und unterschiedliche theoretische und methodische Perspektiven einnehmen (für einen Überblick siehe z. B. Bair 2010). Dabei sind u. a. regionale und sektorale Unterschiede, aber auch Unterschiede zwischen Frauen sichtbar geworden, sodass sie nicht mehr generell als eine homogene Gruppe betrachtet werden, die ausschließlich als Opfer von Ausbeutung in Erscheinung tritt (Mohanty 1991). Dass Geschlecht eine Rolle spielt, ist hierbei der gemeinsame Ausgangspunkt, so Bair (2010), während die Fragen nun eher auf das „Wie“ gerichtet werden, also in welcher Weise Geschlecht in die Form kapitalistischer Akkumulation eingelassen ist (ebd., S. 204). Geschlecht und auch andere Kategorien sozialer Ungleichheit sind demnach nicht als statische Konstante in der Funktionsweise kapitalistischer Akkumulation zu verstehen, sondern sie bedingen sich gegenseitig und dies in einer Form, die aus konkreten sozialen Praxen, aus einem komplexen Set von sozialen, politischen und kulturellen Normen entsteht, die außerdem im Spannungsfeld zwischen globalen und lokalen/ regionalen Strukturen stehen (Bair 2010, S. 205).

Gender-Diskriminierung in globalen Wertschöpfungsketten wurde darüber hinaus auch zunehmend von den großen internationalen Organisationen wie OECD, Weltbank und insbesondere von der ILO aufgegriffen. Diese haben stark dazu beigetragen, dass Gender-Ungleichheit vermehrt thematisiert und wahrgenommen wurde. Darin spiegeln sich Gender-Mainstreaming-Politiken, aber auch die zunehmende Erwerbstätigkeit von Frauen. So ist die Frauenerwerbsquote seit den 1990er-Jahren weltweit deutlich gestiegen (obgleich die Erwerbsquote für beide Geschlechter stärker noch für Männer - in den letzten Jahren insgesamt wieder rückläufig ist, siehe ILO 2018a). Diese weibliche Erwerbsarbeit findet zunehmend im Kontext globaler Wertschöpfungsketten statt. So sind etwa ein Drittel der Industrie-ArbeiterInnen im globalen Süden Frauen, in Asien stellen sie fast die Hälfte der Belegschaften (Barrientos et al. 2004). Viele Studien zeigen Frauen als benachteiligte und diskriminierte Gruppe, die stärker von prekären und informellen Beschäftigungsverhältnissen, von Arbeitslosigkeit, Armut und Gewalt betroffen ist. Darin spiegelt sich die Geschlechtersegregation in der Struktur der Wertschöpfungsketten: So sind mehr Frauen im niedrig bezahlten Dienstleistungsbereich sowie in den Zulieferstrukturen zu finden, während Männer häufiger in den direkt exportierenden Unternehmen arbeiten (OECD 2018), in denen eher höhere Bezahlung, bessere Beschäftigungsbedingungen und tarifvertragliche Regelungen zu finden sind. Gerade im Zuge der Auseinandersetzung um ,decent work“ und ,,social upgrading“ wurde Geschlechterdiskriminierung in eine Vielzahl von Hintergrund- und Positionspapieren bzw. in Policy-Programme der genannten internationalen Organisationen aufgenommen, die zwar nicht ausschließlich, aber häufig den eher funktionalistischen und an Governance-Konzepten ausgerichteten Ansätzen folgen. Nichtsdestotrotz zeigen auch diese Studien die Bedeutung der Schnittstellen zu den Bereichen, die Hopkins und Wallerstein bereits skizziert haben. Und sie integrieren auch verstärkt intersektio- 
nale Ansätze - insbesondere im Hinblick auf Kinderarbeit, moderne Sklavenarbeit, Migration und die damit verbundene Kategorie der nationalen Herkunft (ILO 2016).

Ich möchte nun im Folgenden einige wichtige Punkte dieser Debatte genauer beleuchten.

\section{Feminisierung von Arbeitskraft}

Auch wenn die zunehmende Beschäftigung von Frauen in globalen Wertschöpfungsketten zunächst mit den negativen Effekten von Niedriglöhnen, exzessiven Arbeitszeiten, Entrechtung und Gewalt einherging, hatte die Proletarisierung von Frauen im globalen Süden durchaus emanzipatorische Effekte. Ein eigener Lohn erlaubt Frauen in gewissen Grenzen ökonomische Unabhängigkeit von Ehemännern und patriarchalen Familienstrukturen und verleiht ihnen in neuer Weise einen Status als Staatsbürgerinnen, was es zumindest formal erlaubt, bestimmte rechtliche Regelungen z.B. des Arbeits- und Sozialrechts in Anspruch zu nehmen. Zum anderen sind kollektive Organisationsformen und Widerstand, also eine gewisse Handlungsmacht leichter aufzubauen (z. B. Trzeciak und Tuider 2013). Doch vieles deutet darauf hin, dass dies zunächst keinen deutlichen Trend zu mehr Geschlechtergleichstellung zur Folge hatte. Fernández-Kelly stellte schon in der oben erwähnten Studie von 1983 die Frage bzw. infrage, ob die massive Integration von jungen Frauen in die Industriestandorte im globalen Süden tradierte Formen der geschlechtshierarchischen sozialen Arbeitsteilung ändern würde und insofern als Modernisierung zu sehen sei. Tatsächlich weist die geschlechtshierarchische Arbeitsteilung in den privaten Haushalten weltweit eine hohe Persistenz auf (Ferguson 2013). Hinzu kommt, dass sich eine geschlechtshierarchische Arbeitsteilung in fast allen Bereichen globaler Produktionsnetze reproduziert oder sogar vertieft hat, angefangen von geschlechtsspezifischen Tätigkeiten im betrieblichen Arbeitsprozess, den betrieblichen Hierarchien, den Branchen oder den Regionen (De la O 2013). Gemeinsam ist dabei, dass weiblich konnotierte Tätigkeiten in der Regel die besonders niedrig bezahlten, als niedrig qualifiziert deklarierten, ungeschützten und prekären Beschäftigungsverhältnisse meint. Dies folgt einer machtvollen Konstruktion von Geschlechterbildern und Geschlechterrollen, die eine solche Hierarchisierung diskursiv legitimiert. Interessant ist dabei, dass - obwohl die Geschlechterkonstruktionen in verschiedenen Ländern und Regionen unterschiedlich sind - überall sehr ähnliche Stereotypen zu finden sind, wie z. B. die Frauen mit den ,kleinen geschickten Fingern“, die sich angeblich besonders gut für die Montage kleiner elektronischer Komponenten eignen. Oder: Frauen seien fügsamer und geduldiger und daher für emotionale Arbeit, z. B. in Call Centern etc., besser geeignet (Sproll 2016b). Leslie Salzinger (2003) hat eindrucksvoll nachgezeichnet, wie solche Genderkonstruktionen auf der betrieblichen Ebene entstehen und Geschlechterhierarchien gefestigt werden, die aber gleichzeitig von Betrieb zu Betrieb differieren.

Die geschlechtlichen Vorzeichen von Tätigkeiten und Sektoren sind nicht fixiert, sondern wandelbar, aber sie folgen immer einer Logik der Abwertung weiblicher Arbeit. Ein gutes Beispiel hierfür liefert die mexikanische Maquiladora-Industrie, in der ein deutlicher Wandel in der geschlechtlichen Zusammensetzung der Beleg- 
schaften festzustellen ist. Während in den Anfängen (seit den 1960er-Jahren) eine große Mehrzahl weiblicher Arbeitskräfte beschäftigt war, wurde der Männeranteil in der Produktion zusehends größer, insbesondere in den Sektoren, in denen sich höherwertige Produkte mit einem höheren Einsatz von High-Tech-Infrastrukturen entwickelten, wie z. B. in der Elektronikindustrie oder den Autozulieferern der globalen Autokonzerne (De la O 2003). Hier zeigt sich nicht nur die geschlechtliche Konnotation von spezifischen Branchen und von Technik, sondern es lässt sich eine sehr wichtige Lektion daraus ableiten: Die Höherentwicklung der Maquiladora-Industrie in Mexiko ist ein Paradebeispiel für ein gelungenes industrial upgrading. Und es zeigt gleichzeitig, dass social upgrading nicht automatisch aus einem industrial upgrading resultiert, sondern sogar mit einer Verdrängung weiblicher Arbeitskräfte und damit einer Verschärfung von Geschlechterungleichheit einhergehen kann. Dies erklärt sich nicht unbedingt durch höhere Qualifikationsanforderungen, über die Frauen angeblich nicht verfügen würden. Vielmehr sind oftmals die Arbeitsprozesse so organisiert, dass es zu einer Polarisierung kommt. Dies zeigt sich z.B. in der Auto- und Elektronikindustrie, wo den einerseits stark zunehmenden und hochqualifizierten Ingenieursarbeiten weiterhin eine Masse von niedrigqualifizierten und überwiegend weiblichen Produktionsbeschäftigten gegenüberstehen, auch wenn es sich um High-Tech-Produktion handelt. Dies trifft insbesondere auf neotayloristische Arbeitsorganisationen zu, die weit verbreitet sind und als Schlüssel für die Reduktion von Lohnkosten, aber auch die Ausbeutung und Entrechtung von Beschäftigten durch Flexibilisierung auf allen Ebenen gelten können (Sproll 2010; Lüthje et al. 2013). Wir müssen hier von zwei verschiedenen Prozessen ausgehen: zum einen, dass Qualifikation keine objektive Größe, sondern eine Konstruktion ist, welche die Abwertung von weiblicher Arbeit als unqualifiziert und ersetzbar impliziert (Wright 2006). Diese Logik ist in die spezifische Form des Arbeitsprozesses eingelassen. Zum anderen zeigt sich aber ein grundlegender Wandel von Geschlechterverhältnissen, in dem es einen Anpassungsprozess „,nach unten“ zu geben scheint: Der Begriff der Feminisierung von Arbeitskraft, so wie ihn Guy Standing (1999) geprägt hat, bezieht sich deshalb auch auf Männer, die ähnlichen Bedingungen hinsichtlich Niedriglohn, schlechten und prekären Arbeitsbedingungen etc. ausgesetzt sind, wie sie lange Zeit als typisch für Frauen in den Weltmarktfabriken galten. Damit wird ein allgemeiner Prozess der Prekarisierung von Arbeits- und Lebensbedingungen beschrieben, der insgesamt auf eine Zunahme von sozialer Ungleichheit verweist, die im Übrigen nicht auf den globalen Süden beschränkt ist (Sproll und Wehr 2014). Eine solche Entwicklung impliziert weitreichende Folgen für die Veränderung traditioneller Geschlechterarrangements: ein schlecht bezahlter und prekärer Mann kann kein Familien-Ernährer sein; tradierte Vorstellungen von Maskulinität werden zunehmend infrage gestellt (De la O 2013). Dies bedeutet zwar nicht, dass die Mehrfachbelastung von Frauen aufgehoben wird, die nicht nur erwerbstätig sind, sondern auch als Mütter und (Ehe-)Partnerinnen oder Alleinerziehende die Hauptverantwortung für die Haus-, Erziehungs- und Pflegearbeit tragen. Allerdings scheint das geschlechtsspezifische Verhältnis der Aufteilung zwischen Erwerbs- und Hausarbeit neu gemischt zu werden (Barrientos 2019). 


\section{Soziale Reproduktion: Private Haushalte und informelle Beschäftigung}

Hier kommen wir also wieder zur Frage der Organisation der sozialen Reproduktion. Dunaway hat im Anschluss an Hopkins und Wallerstein die Frage nach den versteckten Inputs der privaten Haushalte in die Wertschöpfungsketten aufgeworfen, die von den global produzierenden Unternehmen angeeignet werden. Sie unterscheidet zwischen den ,,verdeckten Subventionen“ durch produktive Tätigkeiten der Haushalte einerseits und der „Externalisierung“ von Kosten in die Haushalte andererseits (Dunaway 2014b, S. 59). Die privaten Haushalte der Beschäftigten versorgen die Unternehmen nicht nur mit den Arbeitskräften und allem, was zu deren Geburt, Erziehung und Erhaltung nötig ist, sondern bringen auch nicht entlohnte produktive Tätigkeiten (z.B. Frauen, die unbezahlt in der Erwerbsarbeit ihrer Männer in der Landwirtschaft mithelfen) sowie informelle heimbasierte Produktionsarbeit (z. B. in der Textil- oder auch der Elektronikindustrie) mit ein. Diese versteckten Inputs haben den Effekt, dass die Lohnkosten niedrig gehalten werden (ebd., S. 60). Tatsächlich ist die Ausbreitung von Niedriglöhnen ein zentrales Merkmal von globalen Wertschöpfungsketten und nach wie vor eine der Hauptstrategien weltweit, um Kosten zu senken. Dies hat eine breite Diskussion über die sozialen Folgen der wachsenden sozialen Ungleichheit ausgelöst, die u. a. auf einer zunehmenden Spreizung der Einkommen beruht: Eine große Zahl von Lohnabhängigen in der Welt kann nicht auf ein existenzsicherndes Einkommen zählen; davon sind Frauen überdurchschnittlich betroffen (ILO 2018b). Doch was bedeutet es, wenn Löhne so niedrig sind, dass sie die Reproduktion von Arbeitskraft nicht ermöglichen? In meiner Forschung in der IT-Industrie in Guadalajara, Mexiko, wurde z. B. deutlich, dass soziale Reproduktion nur über das Netzwerk Familie gelang, zu dem die einzelnen Familienmitglieder in unterschiedlicher Weise beitrugen. Junge Arbeiterinnen der Elektronikindustrie waren trotz ihrer formalen Vollzeiterwerbstätigkeit (die bei guter Auftragslage oftmals weit über eine 40-Stunden-Woche hinausging) nicht in der Lage, sich selbstständig von ihrem Lohn zu ernähren und eine Miete zu bezahlen. Obwohl viele meiner Interviewpartnerinnen sich dies wünschten, waren sie überwiegend nicht in der Lage, aus dem Elternhaus auszuziehen und sich eine städtische Wohnung zu suchen. Ihre wirtschaftliche Unabhängigkeit hielt sie bei der Herkunftsfamilie, die umgekehrt ebenfalls auf ihren Beitrag zum Familieneinkommen angewiesen war (Sproll 2010, S. $260 \mathrm{ff}$.). Ein anderes Beispiel aus meiner Forschung zur IT-Industrie in Brasilien beleuchtet einen weiteren wichtigen Aspekt: Ein etwa 35-jähriger Arbeiter eines multinationalen Elektronik-Kontraktfertigungsunternehmens berichtete mir im Interview, dass er von seinem Lohn als Produktionsarbeiter für hochwertige Telekommunikations-Infrastrukturen nicht überleben konnte. Jeden Vormittag verkaufte er deshalb Erdnüsse mit Schokoladenüberzug als Straßenverkäufer, die seine Mutter in Heimarbeit herstellte, bevor er seine Arbeit in der Spätschicht im Unternehmen aufnahm (ebd., S. 193). Dies zeigt den Haushalt als ein Netzwerk, in dem unterschiedliche Familienmitglieder mit formeller, informeller und reproduktiver Arbeit zum Überleben beitragen. Es ist ein Ort, an dem bezahlte und unbezahlte Arbeit, formelle und informelle Arbeit zusammenspielen, ohne den die Produktion in den Wertschöpfungsketten nicht denkbar wäre. 
Der große Teil der unbezahlten reproduktiven Arbeit wird nach wie vor von Frauen geleistet, obwohl sie als Lohnarbeiterinnen zunehmend in den Arbeitsmarkt (nicht nur in globalen Wertschöpfungsketten) integriert sind. Eine einfache Zuordnung von öffentlicher Erwerbsarbeit als männliche und der heimischen Reproduktionsarbeit als weibliche Sphäre stimmt deshalb nur teilweise (siehe auch Barrientos 2019) - zumal es hier große regionale Unterschiede in kulturellen und geschlechtsspezifischen Normen gibt. Während aber dennoch Kapitalismus ohne diesen Aneignungsprozess unbezahlter Reproduktionsarbeit und nicht vergüteter Produktionsarbeit wohl nicht überlebensfähig wäre, machen die Beispiele deutlich, dass formale und informelle Arbeit weitgehend miteinander verflochten sind, sei es durch Heimarbeit oder Arbeit auf informellen Märkten, die natürlich am unteren Ende der Kette ebenfalls eine große Rolle spielen. Globale Produktionsnetze eignen sich dabei nicht nur den Mehrwert informeller und/oder unbezahlter reproduktiver Arbeit an, sondern sind aktiv an der Herstellung informeller Strukturen beteiligt, die weltweit zunehmen. Der informelle Sektor ist also nicht nur als Auffangbecken für die „Opfer“ von Krisen, d.h. der zyklischen Produktion von Arbeitslosigkeit, von Bedeutung (Chen 2013), sondern inhärenter Bestandteil globaler Wertschöpfung - und muss als solcher deshalb auch theoretisch einbezogen werden.

Schaut man auf die Externalisierung von Kosten an die privaten Haushalte, geht es also längst nicht nur um die Reproduktion von Arbeitskraft, sondern auch um die Folgen für die Form und Existenz der Haushalte selbst. Wilma Dunaway verweist auf die Veränderungen in der Zusammensetzung der Haushalte z. B. durch Migration (in Folge ökonomischer Not), die Verstärkung und Reproduktion von Ungleichheit innerhalb der Familien. Externalisierung bedeutet auch Folgen für die Gesundheit oder die Führung einer Subsistenzwirtschaft aufgrund der Zerstörung der natürlichen Umwelt (Luft, Wasser, Boden) durch die Konzerne, die oftmals Frauen besonders stark treffen (Dunaway 2014b, S. $59 \mathrm{ff}$.).

Wenn man solche Prozesse mit in die Analyse einbezieht, wird deutlich, dass die Vertiefung sozialer Ungleichheit in und durch globale Wertschöpfungsketten eine Krise der sozialen Reproduktion nach sich zieht - die sich ebenfalls auf einer globalen Ebene reorganisiert. Dies wurde insbesondere mit dem Ansatz der „Global Care Chains“ diskutiert (Hochschild 2000; Yeates 2014), der den Blick auf die Migration von Haushaltshilfen und SorgearbeiterInnen für Kinder, Kranke und alte Menschen aus ärmeren in reichere Regionen und Haushalte richtet. Diese hinterlassen wiederum Lücken für die Sorge um Kinder und Angehörige in ihren eigenen Haushalten, sodass entlang eines tiefgreifenden Prozesses der Kommodifizierung der Haus- und Sorgearbeit neue Linien der Ungleichheit entstehen. Dies zeigt zum einen, dass die globale Transformation kapitalistischer Akkumulation nicht losgelöst von der Transformation reproduktiver Prozesse zu verstehen ist und Ungleichheitsprozesse erst vollständig sichtbar werden, wenn beide Sphären in ihrer Verflechtung in den Blick genommen werden. Zum anderen kommen in diesen neuen Ungleichheitsprozessen Differenzen zwischen Frauen zum Ausdruck, die der Zugehörigkeit zu sozialen Klassen, aber auch regionalen, postkolonialen und ethnischen Kategorien folgen. 


\section{Fragmentierung von Wertschöpfung und Arbeitenden - zur Bedeutung einer intersektionalen Perspektive}

Die globale Ungleichheitsforschung ist längst über den Stand der Forschung hinweg, in dem soziale Ungleichheit weitgehend mit sozio-ökonomischer Ungleichheit gleichgesetzt wurde, sodass andere Ebenen und Kategorien wie postkoloniale Konstellationen, Geschlecht, Race/Ethnizität, Alter, sexuelle Orientierung usw. stärker in den Blick genommen wurden (siehe z. B. Fischer und Grandner 2019). Doch bislang gibt es wenig Forschung, die sich mit einer explizit intersektionalen Perspektive auf globale Wertschöpfungsketten bezieht und die benannten Kategorien in ihrer Multidimensionalität und Verflechtung betrachtet. Transnationale Produktion ist dabei ein sehr wichtiger Forschungsbereich, da es sich um ein dynamisches Feld handelt, indem sich viele Achsen ständig neu überkreuzen. Es ist nicht allein die transnationale Ausdehnung, sondern vor allem die Fragmentierung der Wertschöpfung, die durch Verlagerung von Produktionsstandorten in andere Regionen und die damit verbundenen durchgängigen und sehr weit gehenden Prozesse von Outsourcing und Subcontracting entsteht. Dieser Prozess wird von einem hohen Wettbewerbsdruck getrieben, sodass außerdem Flexibilisierung und andere Strategien zur Senkung von Arbeitskosten, wie prekäre Beschäftigung, Teilzeit, informelle Arrangements bzw. Scheinselbstständigkeit etc. dominieren, die zu einer Diversifizierung von Beschäftigungsformen geführt haben (Flecker 2010). Es ist bereits deutlich geworden, dass z. B. Geschlecht solche Prozesse immer mit strukturiert. Organisationale Fragmentierungen ziehen einen Prozess der hierarchischen Segmentation von Arbeitenden nach sich, der Kernbelegschaften, Randbelegschaften, Arbeitende, die gar keinen Beschäftigtenstatus haben wie Freelancer oder informell Arbeitende, Menschen ohne Papiere, Menschen in modernen Sklaven-Verhältnissen usw. voneinander unterscheidet. Dies erzeugt Konkurrenzdruck zwischen Arbeitenden und führt zu Entsolidarisierung, sodass sich spezifische Machtverhältnisse etablieren. Die Fragmentierung und Spaltung von Beschäftigten entlang von Diskriminierung, die auf Geschlecht, Zugehörigkeit zu einer sozialen Klasse oder Kaste oder zu einer Ethnie/Race beruht, ist somit ein wichtiger Teil der Ausbeutungsverhältnisse in globalen Wertschöpfungsketten, in dem Differenzen gezielt genutzt werden. Dies kann z. B. für die Situation von MigrantInnen gezeigt werden: Die Transnationalisierung von Produktion hat ein beispielloses Ausmaß an regionaler und transnationaler Migration ausgelöst. Diese Bewegung folgt oft ehemaligen kolonialen Wegen - d. h. sie spiegelt Strukturen der Ausbeutung, des Rassismus und der Abwertung von Menschen aufgrund ihrer Ethnizität oder Religion wider. Es reicht also keineswegs, Geschlecht in die Analyse einzubeziehen, um die Ungleichheitsdynamik in globalen Wertschöpfungsketten zu verstehen. Es ist darüber hinaus notwendig, auch innerhalb von Ungleichheitskategorien wie Geschlecht zu differenzieren. Nicht alle Frauen, nicht alle MigrantInnen sind unqualifizierte ProduktionsarbeiterInnen in prekären Verhältnissen, sondern sie unterscheiden sich auch bezüglich ihres Beschäftigungsverhältnisses (unbefristet, informell, unbezahlt, Teilzeit etc.), ihrer Position im Betrieb, ihrer Klassen- oder Kastenzugehörigkeit, ihres Alters, ihres rechtlichen Status usw.

Die Nutzung dieser Differenzen durch Unternehmen spiegelt spezifische Machtverhältnisse, die der Form globaler Produktionsnetze und deren Dynamik von Akku- 
mulation zugrunde liegen. Doch dies spielt sich im Rahmen staatlicher Regulation $\mathrm{ab}$, die Ungleichheit institutionalisiert. Der Staat spielt eine zentrale Rolle nicht nur für die Industriepolitik (die in den Mainstream-Ansätzen zu globaler Wertschöpfung richtigerweise hervorgehoben wurde), sondern auch für die Form der Familien- und Geschlechterpolitik, der Arbeitsmarktpolitik (z. B. bezüglich formaler wie informeller oder prekärer Beschäftigungsformen), der Migrationspolitik und (Anti-)Diskriminierungspolitik. Der Staat ist somit - und trotz der wachsenden Macht transnationaler Konzerne - immer noch einer der wichtigsten AkteurInnen für die Steuerung der Form und Dynamik globaler Wertschöpfung. Er legt die Spielregeln fest, auf welche Weise Unternehmen auf geschlechtsspezifische oder migrationsbezogene Ungleichheiten zurückgreifen können, ob legal oder illegal, ob unter Rückgriff auf formelle, informelle oder Sklaven-Arbeitsmärkte. Die Unterschiede in der nationalen Regulation sind dabei natürlich wichtige Treiber für Standortentscheidungen geworden nationale Regulationsformen reichen schon lange nicht mehr aus, um die Explosion sozialer Ungleichheit in bestimmten Grenzen zu halten.

\section{Ausblick}

Die hier skizzierte Perspektive verdeutlicht, was durch die Integration von Ungleichheit und sozialer Reproduktion in den Blick kommt bzw. was umgekehrt ausgeblendet bliebe. Klar ist, dass die strukturellen Veränderungen, die sich abzeichnen, die Notwendigkeit einer solchen Perspektive immer dringender machen. So ist Informalität fast überall auf der Welt auf dem Vormarsch. Mehr als $60 \%$ der weltweit Beschäftigten arbeiten im informellen Sektor und damit mehr als zwei Milliarden Menschen, die ungeschützt unter prekären Bedingungen arbeiten, so eine Studie der ILO (2018c, S. 1, 31). Angesichts des einsetzenden Umbruchs durch Digitalisierung ist zudem, insbesondere durch die rasche Etablierung der Plattformökonomie, mit einem neuen Schub an prekärer und informalisierter Beschäftigung zu rechnen (Berg et al. 2018), während die Debatten über eine mögliche Regulation noch ganz am Anfang stehen.

Hinzu kommt, dass immer neue Beschäftigtengruppen in globale Wertschöpfungsketten integriert werden, die, z. B. durch Migration, aus ihren sozialen Zusammenhängen herausgelöst werden. Kollektive Identitäten lösen sich dabei mehr und mehr auf, insbesondere der Bezug zu einer ,traditionellen“ Klassenidentität schwindet oder differenziert sich weiter aus - nicht nur durch die zunehmende Erwerbsarbeit von Frauen, sondern auch durch die Zerstörung langfristiger und geschützter Beschäftigungsverhältnisse infolge einer tiefgreifenden Prekarisierung von Arbeit und der damit verbundenen Herausforderungen für die Reproduktion. Während dies auf der einen Seite Gewalt in Form von Rassismus, Femiziden usw. antreibt, entstehen auch neue Formen eines emanzipatorischen Widerstands, der sich nicht zuletzt in der Feminisierung von Streikbewegungen zeigt (Artus 2019). Für eine Weiterentwicklung neuer Organisationstrategien, die auch informelle, prekär Arbeitende in ihrer Diversität umfassen und die Durchsetzung eines ,social upgrading“ in einem weiten Sinne ist es notwendig, Ausbeutung durch Spaltungen und Ungleichheit auf die Agenda zu setzen. Dafür braucht es neue Ansätze, die nicht einseitig aus 
dem Globalen Norden kommen und die sich nicht auf männliche, weiße und formelle Beschäftigte konzentrieren, und insbesondere den Wandel im Verhältnis von Produktion und Reproduktion in den Blick nehmen.

Funding Open Access funding enabled and organized by Projekt DEAL.

Open Access Dieser Artikel wird unter der Creative Commons Namensnennung 4.0 International Lizenz veröffentlicht, welche die Nutzung, Vervielfältigung, Bearbeitung, Verbreitung und Wiedergabe in jeglichem Medium und Format erlaubt, sofern Sie den/die ursprünglichen Autor(en) und die Quelle ordnungsgemäß nennen, einen Link zur Creative Commons Lizenz beifügen und angeben, ob Änderungen vorgenommen wurden.

Die in diesem Artikel enthaltenen Bilder und sonstiges Drittmaterial unterliegen ebenfalls der genannten Creative Commons Lizenz, sofern sich aus der Abbildungslegende nichts anderes ergibt. Sofern das betreffende Material nicht unter der genannten Creative Commons Lizenz steht und die betreffende Handlung nicht nach gesetzlichen Vorschriften erlaubt ist, ist für die oben aufgeführten Weiterverwendungen des Materials die Einwilligung des jeweiligen Rechteinhabers einzuholen.

Weitere Details zur Lizenz entnehmen Sie bitte der Lizenzinformation auf http://creativecommons.org/ licenses/by/4.0/deed.de.

\section{Literatur}

Artus, Ingrid. 2019. Frauen*streik! Zur Feminisierung von Arbeitskämpfen. Analysen, Bd. 54. Berlin: Rosa-Luxemburg-Stiftung.

Bair, Jennifer. 2005. Global capitalism and commodity chains: looking back, going forward. Competition \& Change 9(2):153-180. https://doi.org/10.1179/10245905X45382.

Bair, Jennifer. 2010. On difference and capital: gender and the globalization of production. Signs: Journal of Women in Culture and Society 36(1):203-226. https://doi.org/10.1086/652912.

Bair, Jennifer, und Marion Werner. 2011. Commodity chains and the uneven geographies of global capitalism: a disarticulations perspective. Environment and Planning A: Economy and Space 43:988-997.

Barrientos, Stephanie. 2019. Gender and work in global value chains. Capturing the gains? Cambridge: University Press. https://doi.org/10.1017/9781108679459.

Barrientos, Stephanie, Naila Kabeer, und Naomi Hossain. 2004. The gender dimensions of the globalization of production. Working Paper, Bd. 17. Geneva: ILO.

Berg, Janina, Marianne Furrer, Ellie Harmon, Uma Rani, und M. Six Silberman. 2018. Digital labour platforms and the future of work: Towards decent work in the online world. Geneva: International Labour Office.

Chen, Marta Alter. 2013. Informalität, Geschlecht und die globalen Auswirkungen der großen Rezession. In Arbeit in globaler Perspektive. Facetten informeller Beschäftigung, Hrsg. Hans-Jürgen Burchardt, Stefan Peters, und Nico Weinmann, 149-171. Frankfurt a.M.: Campus.

Collins, Jane. 2014. A feminist approach to overcoming the closed boxes of the commodity chains. In Gendered commodity chains. Seeing womens's work and households in global production, Hrsg. Wilma A. Dunaway, 27-37. Stanford: University Press.

De la O, María Eugenia. 2003. El trabajo de las mujeres en la industria maquiladora de México: balance de cuatro décadas de estudio. Debate Feminista 35:31-56.

De la O, María Eugenia (Hrsg.). 2013. Género y trabajo en las maquiladoras en México: Nuevos actores en nuevos contextos. México: Ediciones de la Casa Chata.

Dunaway, Wilma A. 2014a. Introduction. In Gendered commodity chains. Seeing womens's work and households in global production, Hrsg. Wilma A. Dunaway, 1-24. Stanford: University Press.

Dunaway, Wilma A. 2014b. Through the portal of the household. Conceptualizing women's subsidies to commodity chains. In Gendered commodity chains. Seeing Womens's work and households in global production, Hrsg. Wilma A. Dunaway, 55-71. Stanford: University Press.

Elson, Diane, und Ruth Pearson. 1981. Nimble fingers make cheap workers: an analysis of women's employment in third world export manufacturing. Feminist Review 7:87-107. 
Ferguson, Lucy. 2013. Gender, work, and the sexual division of labor. In The Oxford handbook of gender and politics. Oxford Handbooks Online, Hrsg. Georgina Waylen, Karen Celis, Johanna Kantola, und S. Laurel Weldon, 337-362. https://doi.org/10.1093/oxfordhb/9780199751457.013.0013.

Fernández-Kelly, María P. 1983. For we are sold, I and my people: Women and industry in Mexico's frontier. Albany: State University of New York Press.

Fischer, Karin, und Margarete Grandner (Hrsg.). 2019. Globale Ungleichheit. Über die Zusammenhänge von Kolonialismus, Arbeitsverhältnissen und Naturverbrauch. Wien: Mandelbaum.

Flecker, Jörg. 2010. Fragmenting labour: organisational restructuring, employment relations and the dynamics of national regulatory frame-works. Work Organisation, Labour \& Globalisation 4(1):8-23.

Fröbel, Folker, Jürgen Heinrichs, und Otto Kreye. 1977. Die neue internationale Arbeitsteilung. Reinbek: Rowohlt.

Gereffi, Gary. 1996. Global commodity chains: new forms of coordination and control among nations and firms in international industries. Competition \& Change 1(4):427-439.

Gereffi, Gary. 1999. International trade and industrial upgrading in the apparel commodity chain. Journal of International Economics 48(1):37-70.

Gereffi, Gary, und Raphael Kaplinski. 2001. Value of value chains. IDS Bulletin https://doi.org/10.1111/j. 1759-5436.2001.mp32003001.x.

Gereffi, Gary, und Miguel Korzeniewicz (Hrsg.). 1994. Commodity chains and global capitalism. London: Praeger.

Henderson, Jeffrey, Peter Dicken, Martin Hess, Neil Coe, und Henry Wai-Chung Yeung. 2002. Global production networks and the analysis of economic development. Review of International Political Economy 9(3):436-464.

Hochschild, Arlie R. 2000. Global care chains and emotional surplus value. In On the edge: living with global capitalism, Hrsg. Will Hutton, Anthony Giddens, 130-146. London: Jonathan Cape.

Hopkins, Terence, und Immanuel Wallerstein. 1977. Patterns of development of the modern world-system. Review 1(2):111-145.

Humphrey, John, und Hubert Schmitz. 2002. How does insertion in global value chains affect upgrading in industrial clusters? Regional Studies 36(9):1017-1027.

International Labour Office. 2016. Decent work in global supply chains. Report IV, International Labour Conference, 105th Session, 2016. Geneva: ILO.

International Labour Office. 2018a. World employment and social outlook: trends for women 2018-global snapshot. Geneva: ILO.

International Labour Office. 2018b. Global wage report 2018/19: What lies behind gender pay gaps. Geneva: ILO.

International Labour Office. 2018c. Women and men in the informal economy: a statistical picture, 3. Aufl., Geneva: ILO.

Lipietz, Alain. 1987. Mirages and miracles. The crisis of global Fordism. London: Verso.

Lüthje, Boy, Stefanie Hürtgen, Peter Pawlicki, und Martina Sproll. 2013. From Silicon Valley to Shenzhen. Global production and work in the IT-industry. Lanham: Rowman \& Littlefield.

Mies, Maria. 2014. Patriarchy and accumulation on a world scale. Women in the international division of labour. London: Zed Books.

Mohanty, Chandra Talpade. 1991. Under western eyes: feminist scholarship and colonial discourses. In Third world women and the politics of feminism, Hrsg. Chandra Talpade Mohanty, Ann Russo, und Lourdes Torres, 51-80. Bloomington: Indiana University Press.

Nash, June, und Maria P. Fernández-Kelly. 1983. Women, men, and the international division of labor. Albany: SUNY Press.

OECD. 2018. Women in global value chains. https://www.oecd.org/sdd/its/Women-in-GVCs.pdf. Zugegriffen: 22. März 2020.

Ong, Aihwa. 1987. Spirits of resistance and capitalist discipline: factory women in Malaysia. Albany: SUNY Press.

Roth, Julia. 2019. Globale Achsen der Ungleichheit: Intersektionalität und/als verflochtene Machtverhätnisse. In Globale Ungleichheit. Über die Zusammenhänge von Kolonialismus, Arbeitsverhältnissen und Naturverbrauch, Hrsg. Karin Fischer, Margarete Grandner, 169-183. Wien: Mandelbaum.

Salzinger, Leslie. 2003. Genders in production: making workers in Mexico's global factories. Berkeley: University of California Press.

Selwyn, Ben. 2012. Beyond firm-centrism: Re-integrating labour and capitalism into global commodity chain analysis. Journal of Economic Geography 2012(12):205-226. https://doi.org/10.1093/jeg/ lbr016. 
Sproll, Martina. 2010. High-Tech für Niedriglohn: Neotayloristische Produktionsregimes in der IT-Industrie in Brasilien und Mexiko. Münster: Westfälisches Dampfboot.

Sproll, Martina. 2016a. Globale Arbeit und Produktion. In Handbuch Entwicklungsforschung, Hrsg. Karin Fischer, Gerhard Hauck, und Manuela Boatcă, 249-260. Wiesbaden: Springer VS.

Sproll, Martina. 2016b. Missing links in service value chain analysis-Space, identity and inequality in Brazilian call centres. In Space, place and global digital work, Hrsg. Jörg Flecker, 105-125. Basingstoke: Palgrave Macmillan.

Sproll, Martina, und Ingrid Wehr (Hrsg.). 2014. Capitalist peripheries: perspectives on precarisation from the Global South and North. Journal für Entwicklungspolitik, Bd. 2014(4) Special Issue.

Standing, Guy. 1999. Global feminization through flexible labor: a theme revisited. World Development 27(3):583-602.

Taylor, Phil, Kirsty Newsome, und Al Rainnie. 2013. Editorial: putting labour in its place: global value chains and labour process analysis. Competition and Change 17(1):1-5.

Trzeciak, Miriam, und Elisabeth Tuider. 2013. Zwischen Ausbeutung und Empowerment? Genderspezifische Handlungsmacht von Maquiladora-Arbeiterinnen in Nordmexiko. In Wanderungen. Migrationen und Transformationen aus geschlechterwissenschaftlichen Perspektiven, Hrsg. Annika McPherson, Barbara Paul, Sylvia Pritsch, Melanie Unseld, und Silke Wenk, 71-87. Bielefeld: transcript.

Wright, Melissa. 2006. Disposable women and other myths of global capitalism. New York: Routledge.

Yeates, Nicola. 2014. Global Care Chains. Bringing in transnational reproductive laborer households. In Gendered commodity chains. Seeing womens's work and households in global production, Hrsg. Wilma A. Dunaway, 175-189. Stanford: University Press.

Martina Sproll ist Professorin für Gesellschaftswissenschaften an der HWR Berlin. Ihre Hauptforschungsgebiete sind Transnationale Produktion und Arbeit, Geschlechterverhältnisse, intersektionale soziale Ungleichheit, politische Ökonomie Lateinamerikas und Europas, Digitalisierung von Arbeit. 